\title{
On an improved method for solving evolution equations of higher order importantly in science and technology
}

\author{
A. Huber ${ }^{1}$ \\ ${ }^{1}$ A-8062 Kumberg, Prottesweg 2a, AUSTRIA \\ e-mail: soliton.alf@web.de
}

\begin{abstract}
In this paper we introduce a new algebraic procedure to compute new classes of solutions of (1+1)-nonlinear partial differential equations (nPDEs) both of physical and technical relevance. The basic assumption is that the unknown solution(s) of the nPDE under consideration satisfy an ordinary differential equation (ODE) of the first order that can be integrated completely. This solution manifold of these first-order ODEs play an essential part in solving given nPDEs. A further important aspect however is the fact that we have the freedom in choosing some parameters bearing positively on the algorithm and hence, the solution-manifold of any nPDEs under consideration are therefore augmented naturally. The present algebraic procedure can widely use to study many nPDE and is not only restricted to time-dependent problems. We note that no numerical methods are necessary and so analytical closed-form classes of solutions result. The algorithm works accurately, is clear structured and can be converted in any computer language. On the contrary it is worth to stress the necessity of such sophisticated methods since a general theory of nPDEs does not exist.
\end{abstract}

Keywords: Nonlinear partial differential equations, evolution equations, special function methods.

PACS-Code: 02.30Jr, 02.20Qs, 02.30Hq.

\section{Introduction}

This article introduces an important concept in the study of nPDE especially of higher order. The important outcome is that one is able to derive classes of solutions by an algebraic approach. In what follows we suppress the item 'classes'; so 'classes of solutions' are simply 'solutions'.

Many models in physics and further technical applications can be described by nPDEs of the general form $u_{t}=K\left[u, u_{x}, u_{x x}, \ldots.\right]$, where $K[u]$ is a nonlinear operator in general (higher order time derivatives are also considered).

Explicit solutions are of basic interest especially those with physical relevance, e.g. the propagation of traveling waves. It is still of interest to evaluate new or improve known methods for finding analytical closed-form solutions since the calculation of the general solution-manifold fails.

Many powerful methods such as the inverse scattering transform method (Drazin and Johnson, 1989), the Darboux transformation (Drazin and Johnson, 1989; Wadati and Konno, 1975), Hirota's bilinear method (Drazin and Johnson, 1989; Wadati and Konno, 1975), the Painlevé expansion (Cariello and Tabor, 1989) and the Homogeneous Balance Method, (HBM), (Wang, 1996) are appropriate to handle such problems efficiently.

The Jacobian elliptic function method described in (Cao, 2001) and (Chen and Zhang, 2003) is also suitable to calculate solutions and a technique using series of sine- and cosine functions is discussed in (Bai, 2001). Derivation and discussions of more analytical solutions are listed in (Elwakil and El-labany, 2003; Elwakil and El-labany, 2004a,b).

Further applications of algebraic approaches were performed in (Huber, 2005) in which solutions of any nPDEs under consideration are assumed in terms of hyperbolic tangent functions. 
A generalized approach; the so-called improved projective Riccati Equation method is derived in (Chen, 2003) as well as the Weierstrassian elliptic function method (Huang, 2004). In recent papers, (Huber, 2006; Huber, 2008), we applied the method both to a combined KdV-mKdV Equation and moreover, an evolution equation of the fourth order was solved for the first time successfully; for a new application of this nPDE, see (Huber, 2010). For further reading 'classical ansatz-methods' are discussed intensively in Darwish (2007), Khuri (2007) and Nickel (2007).

Similarity reductions dealing with Lie symmetries and invariant properties are also appropriate to calculate solutions as shown by the author’s recent papers, e.g. (Huber, 2008; Huber, 2009).

\section{Description of the method}

Consider a given nPDE in its two variables $x$ and $t$ which describes the dynamical evolution of a wave form $u(x, t)$, $u: \Omega \rightarrow R$ in the domain $\Omega \subseteq R^{d}, d \geq 2$ and $t$, so that the mapping holds for $u: \Omega \times R^{+} \rightarrow R, R^{+}=\{t \in R, t>0\}, \Omega \subseteq R^{d}$ :

$$
P\left(u, \frac{\partial u}{\partial x}, \frac{\partial u}{\partial t}, \frac{\partial^{2} u}{\partial x^{2}}, \frac{\partial u}{\partial x} \frac{\partial u}{\partial t}, \ldots \ldots, \frac{\partial^{n-1} u}{\partial x^{n-1}}, \frac{\partial^{n} u}{\partial x^{n}}\right)=0
$$

Firstly the nPDE is converted into a nonlinear ordinary differential equation (nODE) by $u(x, t)=f(\xi), \xi=x-\lambda t$ and $\lambda$ is a constant to be determined later (in general wave theory or solitary propagation $\lambda$ means the velocity).

Thus,

$$
P\left[f(\xi), f^{\prime}(\xi), f^{\prime \prime}(\xi), \ldots . .\right]=0,
$$

with the prime denoting differentiation w. r. t. the independent variable $\xi . f(\xi)$ represents a localized wave solution and exemplifies a stationary wave with characteristic width $L=\lambda^{-1}$.

Note: Considering the traveling wave reduction $\xi=x-\lambda t$ it sometimes might be useful to introduce a constant $\alpha$ so that we have $\xi=\alpha x-\lambda t$; but here we will not follow this intension.

The nODE (2.2) is integrated as long as all terms contain derivatives. Further the associated integration constants can taken to be zero in view of the localized solution one is looking for.

This is a necessary (but not sufficient) condition that $f(\xi)$ tend to zero for $\xi \rightarrow \pm \infty$.

The next step is that the solution can be expressed in terms of the following series representation by using an auxiliary variable $\omega=\omega(\xi)$ such that

$$
f(\xi)=f(\omega(\xi))=a_{0}+\sum_{i=1}^{n} \cosh ^{i-1}\{\omega(\xi)\}\left[a_{i} \sinh (\omega(\xi))+b_{i} \cosh (\omega(\xi))\right]
$$

Since the functional dependence of the function $\omega$ should be clear we drop the argument. The balancing parameter $n$ in the series expression (2.3) is found by balancing the highest-order nonlinear term with the highest-order partial derivative term in the relevant equation (2.2). In case of fractions one can take transformations as shown in Example 2 later.

In addition, and this is the new crucial step it is assumed that the function $\omega$ satisfies the following classes of first-order ODEs (solely the plus sign will be used):

Case A: $\quad \frac{d \omega}{d \xi}=\omega^{\prime}=\alpha \pm \beta \sinh \omega, \omega: R \times R \rightarrow R, \omega \neq 0, \omega^{\prime} \neq 0, \xi \in R, \omega \in R$,

Case B: $\quad \frac{d \omega}{d \xi}=\omega^{\prime}=\alpha \pm \beta \cosh \omega, \omega: R \times R \rightarrow R, \omega \neq 0, \omega^{\prime} \neq 0, \xi \in R, \omega \in[1,+\infty]$,

Case C: $\quad \frac{d \omega}{d \xi}=\omega^{\prime}=\alpha \pm \beta \sinh \omega \cosh \omega, \omega: R \times R \rightarrow R, \omega \neq 0, \omega^{\prime} \neq 0, \xi \in R, \omega \in R \cup[1,+\infty]$.

We assume existence and uniqueness at least of the r.h.s. of the given ODEs and $\alpha$ and $\beta$ are some coefficients determined later and may not vanish commonly.

We summarize all solutions which can be verified by direct integration: 
Case A: $\omega= \pm 2 \arctan h\left[\frac{\beta \mp \sqrt{-\alpha^{2}-\beta^{2}} \tan \left[\frac{1}{2} \sqrt{-\alpha^{2}-\beta^{2}} \xi\right]}{\alpha}\right], \quad \alpha \neq 0, \omega \in R,|\xi|<1$,
Case B: $\omega=-2 \arctan h\left[\frac{\sqrt{-\alpha^{2} \mp \beta^{2}} \tan \left[\frac{1}{2} \sqrt{-\alpha^{2} \mp \beta^{2}} \xi\right]}{\alpha-\beta}\right], \quad \alpha=\beta \neq 0, \omega \in R,|\xi|<1$,
Case C: $\omega=\arctan h\left[\frac{-\beta-\sqrt{-4 \alpha^{2}-\beta^{2}} \tan \left[\frac{1}{2} \sqrt{-4 \alpha^{2}-\beta^{2}} \xi\right]}{2 \alpha}\right], \quad \alpha \neq 0, \omega \in R,|\xi|<1$.

Some constants of integration are dropped; they do not influence the result in a great manner (some shifts might be possible). Now substitute (2.4), (2.4.a) or (2.4.b) together with (2.3) into the nODE (2.2) and change it into polynomial identities for the variable $\omega$ by using the standard relations for the hyperbolic functions.

Then all terms with the same power in $\cosh ^{k} \omega \sinh ^{l} \omega, k, l=0,1, \ldots$ are collected and set to zero their coefficients to arrive a nonlinear algebraic system of polynomial equations solving for the unknowns $\lambda, a_{0}, \ldots, a_{n}, b_{1}, \ldots ., b_{n}$ as well as $\alpha$ and $\beta$ in a consistent way. At this stage it may happen that the solution is of trivial form only and therefore useless for the solution-manifold of eq.(2.1).

Let us take a few words discussing some general remarks: Algebraic methods can widely use for solving nPDEs especially of higher order. In engineering sciences as well as any technical applications solutions of nPDEs are of fundamental interest. Solution techniques should work fast and easy without studying the complicate theory of nonlinear equations.

Another benefit is the fact that we do not need any numerical methods (in some cases it seems that the relating nODE derived from the given nPDE could solved only by numerical methods).

In addition we have the possibility to convert the procedure in any computer languages at once and furthermore we point out that some packages yet exist (Parkes and Duffy 1997; Parkes and Duffy 1996).

The crucial step however covers the following question: Can we us arbitrary ODEs like eq.(2.4), eq.(2.4.a) and eq.(2.4.b) ?

This question has to negate in general since the ODEs have to satisfy some requirements:

(i) They have to be of the first order. (ii) The explicit solution representation has to be known explicitly.

(iii) In case of implicit solutions we have to ensure that the independent variable is expressible definitely. (iv) In case of equations of the form $P\left(\xi, f, f^{\prime}, f^{\prime 2}\right)=0$ we require that by using algebraic operations no root expressions occur. (v) We both require existence (e.g. Peano is sufficient) and uniqueness of the solution(s). (vi) In case of an explicit representation, say, $\omega^{\prime}=f(\xi, \omega)$, the function $f(\xi, \omega)$ has special properties which simplify the integration of the equation $\omega^{\prime}=f(\xi$, $\omega)$. This was the reason for introducing Riccati Equation(s) in the past where the structure of the function $f(\xi, \omega)$ was sufficiently simple.

Summarizing these facts we conclude that the repertoire of ODEs of the first-order is certainly restricted.

Another point of interest is the series, eq.(2.3). Several combinations of the functions involved are therefore thinkable.

On the other hand if the balancing parameter vanishes all algebraic methods fail. We confess this lack but sometimes suitable chosen transformations will help.

In some cases we are interested in complex-valued solutions of eq.(2.1) so we have to extend the domain. For the ODEs eq.(2.4), eq.(2.4.a) and eq.(2.4.b) we require:

Let $D$ be a complex domain and $D \subseteq C \times C$ for all holomorphic functions and further we assume $\xi: C^{\infty} \times C^{\infty} \rightarrow C^{\infty}$ so that we have $\left\{\omega, \omega^{\prime}, \omega^{\prime \prime}, \ldots ., \omega^{(n)}\right\} \neq 0$ and the prime means $d / d \xi$.

In the following we study some selected examples of important equations to clarify the new approach where the first example is performed in detail. For the remaining cases we only present the results. 


\section{Examples of various nPDEs}

\section{Example 1: The viscous Burgers Equation (BE)}

We start by discussing the well-known viscous Burger Equation (Burgers, 1948; Burgers, 1974). A lot of papers and text books cover this equation whereby the equation plays an important role in fluid dynamics with $\mu>0$ as the viscosity parameter

$$
\mu \frac{\partial^{2} u}{\partial x^{2}}=u \frac{\partial u}{\partial x}+\frac{\partial u}{\partial t}, u=u(x, t), u \in R^{2}(-\infty, \infty), t>0 .
$$

Eq.(3) is a simplified model for turbulence, boundary layer behaviour, shock wave formation and mass transport. Several new solutions by considering new approaches developed by the author are available (Huber, 2009). By using a frame of reference $u(x, t)=f(\xi), \xi=x-\lambda t$, the balancing parameter $n$ is determined to be $n=1$. This leads to the next step that, therefore, the polynomial 'ansatz' $\propto a_{0}+a_{1} \sinh \omega+b_{1} \cosh \omega$ is suitable. We derive the following nonlinear algebraic polynomial system for the unknowns $a_{0}, a_{1}, b_{1}, \alpha, \beta$ :

$$
\begin{aligned}
& a_{0} a_{1}-a_{1} \lambda-b_{1} \alpha \mu=0, \\
& a_{1}^{2} \alpha+b_{1}^{2} \alpha+a_{0} a_{1} \beta-a_{1} \beta \lambda-3 b_{1} \alpha \beta \mu=0, \\
& a_{0} b_{1} \alpha+a_{1} b_{1} \beta-b_{1} \alpha \lambda-a_{1} \alpha^{2} \mu-b_{1} \beta^{2} \mu=0, \\
& 2 a_{1} b_{1} \alpha+a_{0} b_{1} \beta-b_{1} \beta \lambda-3 a_{1} \alpha \beta \mu=0, \\
& a_{1}^{2} \beta+b_{1}^{2} \beta-2 b_{1} \beta^{2} \mu=0, \\
& a_{1} b_{1}-a_{1} \beta \mu=0 .
\end{aligned}
$$

In total we have seven solutions whereby five are of trivial form. Useful solutions for the unknown parameters are given

$$
a_{0}=\mp \frac{b_{1} \alpha}{\beta}+\lambda, \quad a_{1}=\mp b_{1}, \quad b_{1} \neq 0, \quad \beta \neq 0 .
$$

Note: We also found a solution including in the trivial case by $a_{0}=\lambda, a_{1}=-b_{1}, \alpha=0$; this case covers earlier known results. Now by using the solution eq.(2.5), Case A, we obtain formally new solutions of the Burgers Equation in the form

$$
f(\xi)=\frac{1}{\beta}\left\{\beta \lambda-b_{1} \alpha-\frac{b_{1} \beta\left(\beta-\alpha-P \tan \left[\frac{P \xi}{2}\right]\right)}{\alpha+\beta P \tan \left[\frac{P \xi}{2}\right]}\right\}, \quad P=\sqrt{-\alpha^{2}-\beta^{2}} .
$$

The function is not defined over the domain where the denominator vanishes. By choosing special values, say, $b_{1}=\lambda=1$ and $\alpha=2, \beta=3$ we get a new solution of the form

$$
f(\xi)=\frac{1}{3}\left[1-\frac{3\left(1+\sqrt{13} \tanh \left[\frac{\sqrt{13}}{2} \xi\right]\right.}{5+\sqrt{13} \tanh \left[\frac{\sqrt{13}}{2} \xi\right]}\right], \quad \xi=x-t
$$

A graphical overview by using some selected values for the parameters is given in Figure 1. It is worth to stress that by using the new approach both the kink- and antikink solutions result 


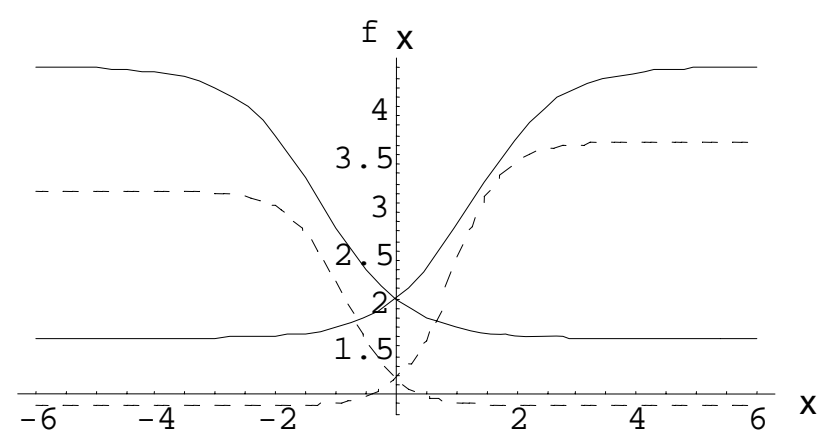

Fig.1 Some special solution curves of the eq.(3.4) representing kink and antikink-like behaviour. Solid curve for the kink solution: $\alpha=\beta=-1$ and $\alpha=-1, \beta=-2$ for the antikink, dotted curve for the kink: $\alpha=\beta=1$ and for the antikink $\alpha=1, \beta=2$.

Note, that like the Sine Gordon Equation, this solution is not of solitary type. For all cases the velocity is assumed to be the unity velocity $\lambda=1$.

Some important properties of the solution should be mention: On the contrary to the Sine Gordon Equation, e.g. which allows traveling kink-solutions, here, we have no traveling solitary motion since the boundary conditions are not fulfilled.

That means that this traveling solution does not vanish rapidly as $|\xi| \rightarrow \infty$. The limiting behaviour is seen to be finite either for the cases $|\xi| \rightarrow \infty$ and $|\xi| \rightarrow 0$.

In the considered domain the function is stable and has a continuous profile. The given analysis was performed if we assume that the viscosity parameter does not influence the system of nonlinear algebraic polynomial equations, eq.(3.1).

If we consider, however, the viscosity parameter as a further unknown we also have seven solutions in total with two of trivial form. We chose

$$
a_{0}=\lambda-\alpha \mu, a_{1}=-b_{1}, \beta=b_{1} / \mu, b_{1} \neq 0, \mu \neq 0
$$

to derive solutions of the general form

$$
f(\xi)=\lambda-\alpha \mu+\frac{\mu\left(\mu-1-R \tan \left[\frac{R \xi}{2}\right]\right)}{\mu-1+R \tan \left[\frac{R \xi}{2}\right]}, \quad R=\sqrt{-\alpha^{2}-\frac{1}{\mu^{2}}} .
$$

On the contrary to the solution eq.(3.3) the viscosity parameter influences the solution significantly.

The solution vanishes on the singularities of the denominator, so we have to require that we exclude those values of the domain of definition: $\forall \xi \backslash\left\{\xi= \pm \frac{1}{R} \arccos [\sqrt{(-1 / \mu-\mu) / 2}]\right\}$.

In Figure 2 we compare two cases of the solution, eq.(3.6). Firstly by choosing positive values for the constants, say $\alpha=b_{1}=\lambda=1$ we also get kink-like profiles.

The profile decreases monotonically to a constant value as $|\xi| \rightarrow \infty$. Otherwise, by choosing negative values, that is $\alpha=b_{1}=\lambda=-1$, singular unstable solutions occur. 

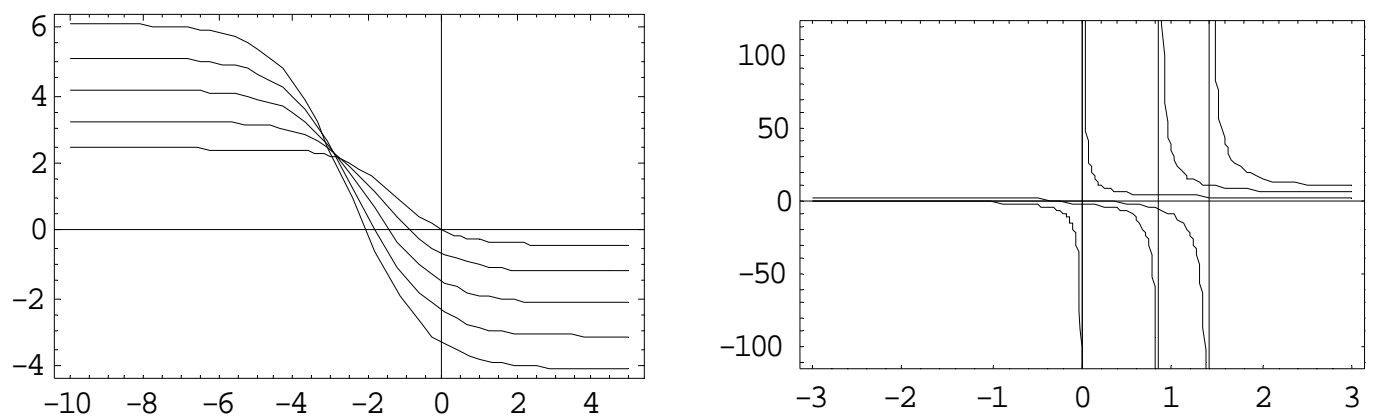

Fig. 2 Solution curves influenced by the viscosity parameter $\mu$, eq.(3.6). Left: Stable kink-like solutions generated by $0<\mu<5$ and $\alpha=\beta=b_{1}=1$. Right: Unstable singular solutions by $1<\mu<3$ and $\alpha=\beta=b_{1}=-1$.

\section{Example 2: A nonlinear reactions-diffusions equation with reaction term:}

An example of practical interest is known as the nonlinear reaction-diffusions equation with a reaction term, e.g. (Whilemsson, 1987). In general one can assume for such classes of equations the form of a conservation equation in three space dimensions so that

$$
\frac{\partial u}{\partial t}+\nabla \vec{F}=f(\vec{x}, t, u)
$$

where $\vec{F}$ is a general flux transport due to diffusion and $f(\vec{x}, t, u)$ is the source or reaction term. For the case of general diffusion problems we can take $\vec{F}=-\kappa \nabla u$, so that eq.(3.7) becomes

$$
\frac{\partial u}{\partial t}=\nabla(\kappa \nabla u)+f(\vec{x}, t, u),
$$

and $\kappa=\kappa(\vec{x}, u)$ is a function of $x$ and $u$. For the case of several chemicals or interacting species, the vector $u_{i}(\vec{x}, t), i=1, \ldots . ., n$, represents concentrations or densities each diffusing with its own diffusion coefficient $\kappa_{i}$ and interacting according to the vector source term $f(\vec{x}, t, u)$. In the one-dimensional case with $f$ as a function of $u$ only, we can write the eq.(3.7a) as

$$
\frac{\partial u}{\partial t}=\frac{\partial}{\partial x}\left[\kappa(u) \frac{\partial u}{\partial x}\right]+f(u) .
$$

If we assume a general polynomial dependence upon the function $f(u)$ we have $f(u)=\alpha u^{m}\left(1-u^{n}\right)$, where $\alpha, m$ and $n$ are some positive constants.

For a suitable choice of the constants and introducing non-vanishing parameters $p$ and $q$ we derive at the following evolution equation

$$
u_{t}-\frac{1}{2}\left(u^{2}\right)_{x X}=p u-q u^{2}, \quad u=u(x, t), \quad t \in R^{+} .
$$

Several finite difference methods have been suggested elsewhere even for problems involving complex geometries, anisotropic materials and time-dependent boundary conditions.

Some results can be found on the similarity solution to heat transfer through extended surfaces especially variable thermal properties.

We seek for solutions for which $u=F(x, t), F \in C^{2}(D)$ and $D \in R^{2}$ with $p \neq 0, q \neq 0$. Using a frame of reference by setting $u(x, t)=f(\xi), \xi=x-\lambda t$ we derive at

$$
\lambda f^{\prime}-\left(f^{2}\right)^{\prime \prime}-p f+q f^{2}=0
$$

This is an example in which the balancing number is not a positive integer $(n=-1)$ and hence, a transformation is necessary.

Let $f=V^{-1}$, then eq.(3.8) transforms into 


$$
2 V V^{\prime \prime}-p V^{3}-6 V^{\prime 2}+V^{2}\left(q-\lambda V^{\prime}\right)=0, \quad V=V(\xi)
$$

Balancing $V^{2} V^{\prime}$ and $V V^{\prime \prime}$ gives $n=1$ and the polynomial 'ansatz' $\propto a_{0}+a_{1} \sinh w+b_{1} \cosh w$ is suitable for considering e.g. the Case B.

Remark: At this stage let us point out an important fact. By balancing the eq.(3.8), $n=-1$ is not the only choice since the procedure also allows the condition $n=-2$ which is missed in several studies. As a further new result we perform the calculation exactly for this case leading to new results.

For the case $n=1$ we deduce: The nonlinear algebraic system consists of eight equations for six unknown, therefore the system is over-determined. If we consider the case that the parameters $p$ and $q$ do not involve in the nonlinear algebraic system we get 19 solutions in total with five solutions of trivial character (we also have to exclude the case $\alpha=\beta$ which appears naturally).

For demonstration we choose the combination

$$
a_{1}=b_{1}, \quad \alpha=\frac{\left(a_{0}^{2}+b_{1}^{2}\right) \beta}{2 a_{0} b_{1}}, \lambda=-\frac{\beta}{b_{1}}, \quad a_{0} \neq 0, \quad b_{0} \neq 0,
$$

and new solutions of practical interest are given finally

$$
V(\xi)=\frac{\left(\left(a_{0}-b_{1}\right)\left(a_{0}^{2}+2 a_{0} b_{1}-b_{1}^{2}+K \tanh \left[\frac{K \beta}{4} \xi\right]\right)\right)}{a_{0}^{2}+b_{1}^{2} K \tanh \left[\frac{K \beta}{4} \xi\right]}, K=\sqrt{a_{0}^{4}+6 a_{0}^{2} b_{1}^{2}+b_{1}^{4}} .
$$

Now by using a suitable choice for the parameters, say, $a_{0}=2$ and $b_{1}=1$, we get special solutions

$$
f(\xi)=\left(1+2(5+\sqrt{41} \tanh [1 / 8 \sqrt{41} \beta \xi])^{-1}\right)^{-1}
$$

Here we have to exclude values where the function takes singular, that is $\xi \neq-\frac{1}{\sqrt{41}} \arctan \left[\frac{7}{\sqrt{41}}\right]$.

In Figure 3 we compare the solution considering fixed values either for the parameters $\beta$ and $b_{1}$. The function takes a finite value as $|\xi| \rightarrow 0$ and takes infinite as $|\xi| \rightarrow \infty$. In this case typical irregular solutions without any stability occur.
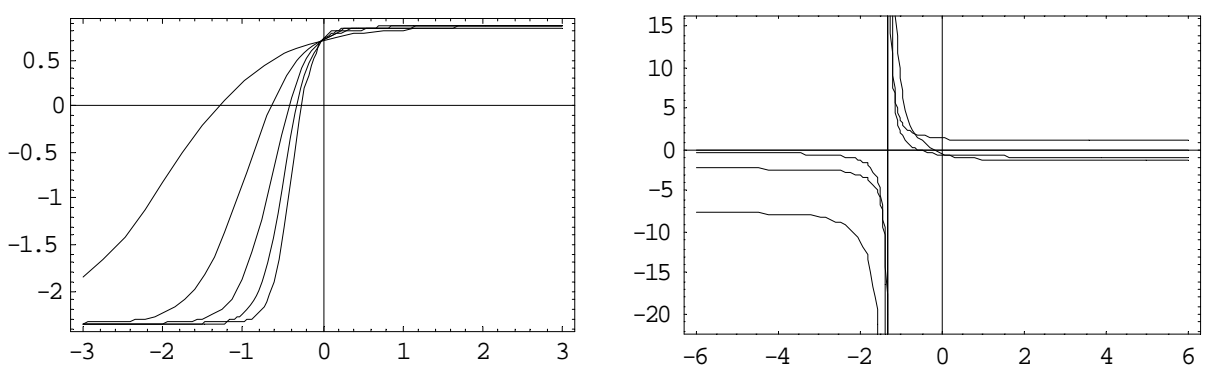

Fig.3 Typical solution curves of the solution eq.(3.11). Left: The parameter $\beta$ varies between $1<\beta<5$. Steep rising kink-like behaviour is seen tending to infinity on both sides. Right: The parameter $\beta$ is assumed to be fix and we chose $1<b_{1}<4$; a typical singular unstable behaviour is remarkable.

If we use the parameters $p$ and $q$ as unknowns in the nonlinear algebraic system the situation changes We have 27 solutions apart from 20 trivial cases which are useless for constructing solutions. For building up new solutions we take the following choice: 


$$
a_{0}=-\frac{\sqrt{4 b_{1} p^{2}+q^{2}}}{2 \sqrt{q}}, \quad \beta=-\frac{b_{1} p}{\sqrt{q}}, \lambda=\frac{p}{\sqrt{q}}, \quad a_{1}=b_{1} .
$$

The analysis leads to the following new types of solutions for the nPDE, eq.(3.7)

$$
f(\xi)=\frac{2 p\left(\sqrt{4 b_{1} p^{2}+q^{2}}-L-q \sqrt{q} \tan [1 / 4 L \xi]\right)}{\left(q+\sqrt{4 b_{1} p^{2}+q^{2}}-2 p\right)(2 p+q-L \tan [1 / 4 L \xi])}, \quad L=\sqrt{-\frac{8 p^{2}}{q}} .
$$

Here we have to require that the parameter q takes negative to ensure the positivity of the root. Let us now handle the second case with $n=-2$.

Applying the procedure we derive at the nODE

$$
V V^{\prime \prime}-p V^{3}-3 V^{\prime 2}+V^{2}\left(q+\lambda V^{\prime}\right)=0, V=V(\xi),
$$

which differs from the nODE (3.9).

Let the new transformation be $V(\xi)=\vartheta^{-2}(\xi)$ and by using the hyperbolic 'ansatz' of the second order a long-winded calculation gives a possible parameter choice

$$
\begin{aligned}
a_{1}= & -\frac{4 \sqrt{3} \beta-15 \sqrt{3} b_{1} p-84 \sqrt{3} \alpha \beta-24 \beta \lambda}{27 p}, a_{2}= \pm \frac{4 \beta^{2}}{\sqrt{3} p}, b_{2}=-\frac{4 \beta^{2}}{p}, \\
a_{0} & =-\frac{1}{5346 p \beta^{2}}\left[114 b_{1} p \beta^{2}-477 b_{1}^{2} p^{2}-3582 b_{1} p \alpha \beta-720(\alpha \beta)^{2}+\right. \\
& \left.+1452 \alpha \beta^{3}-5552 \beta^{4}-66 \sqrt{3}\left(b_{1} p \beta \lambda+10 \alpha \beta^{2} \lambda\right)+68 \sqrt{3} \beta^{3} \lambda+120(\beta \lambda)^{2}\right],
\end{aligned}
$$

where the parameters $b_{1}, \alpha, \beta$ and $\lambda$ can be chosen arbitrary.

Avoiding a duplication of effort in calculating the function $\vartheta(\xi)$ we restrict the analysis to the following special case by requiring $\beta=\lambda=b_{1}=p=q=1$ and $\alpha=-1$ so that some roots may not vanish.

In total a completely new solution function is derived allowing to augment the solution-manifold in the form

$$
\begin{aligned}
\vartheta(\xi) & =2 \cosh [2 \ln \Phi]+\frac{2}{\sqrt{3}} \sinh [2 \ln \Phi]+\frac{3+7 \cosh \xi+2 \sqrt{2} \sinh \xi}{5 \cosh \xi-2 \sqrt{2} \sinh \xi}+ \\
& +\{4(73 \sqrt{3}-24)(1+\sqrt{2} \operatorname{tgh}[\xi / 2])\}\{27(2(\sqrt{2}+\operatorname{tgh}[\xi / 2]-3))\}^{-1},
\end{aligned}
$$

where we used the abbreviation $\Phi=2 \ln \left[\frac{4}{1-\sqrt{2} \tanh [\xi / 2]}-1\right]$ together with some basic transformations for the hyperbolic and inverse trigonometric functions.

To be a unique solution we have to exclude some values for the argument. The function is defined for all $\xi_{1} \neq 2 \tanh ^{-1}[-3 / \sqrt{2}]$ and $\xi_{2} \neq 2 \tanh ^{-1}[-1 / \sqrt{2}]$ and for the inverse trigonometric functions as usual on $D_{f} \in R \backslash\{-1,1\}$.

The function $\vartheta(\xi)$ itself as well as the first and the second derivatives are finite and positive functions at the point $\xi=0$.

The limit calculations show that both for the cases $|\xi| \rightarrow 0$ and $|\xi| \rightarrow \pm \infty$ the function remains finite.

A graphical overview is given in Figure 4 whereby a typical peakon solution is remarkable.

Note: It is sufficient to consider only the function $\vartheta(\xi)$ since squaring and taking the reciprocal does not influence the final result significantly. 

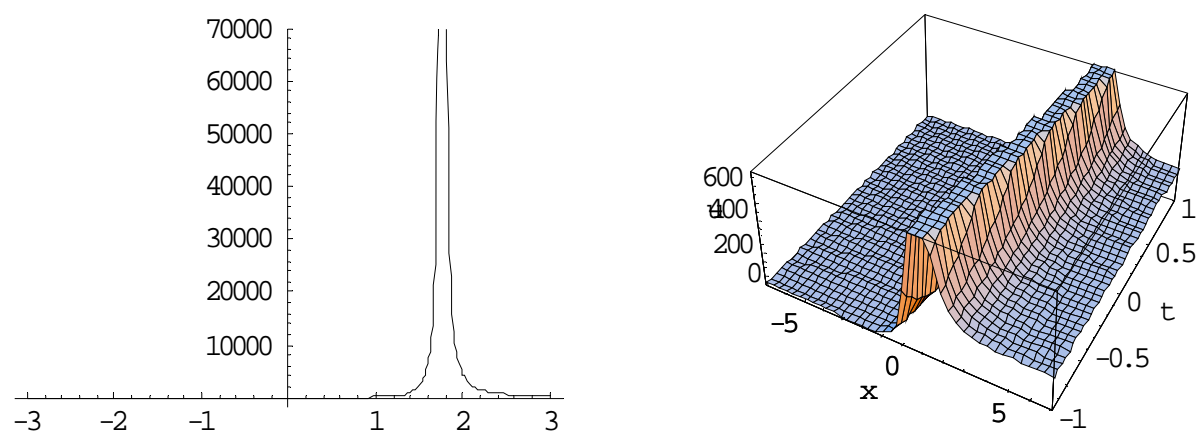

Fig.4 Left: A planar plot of the solution function (3.16) showing a peakon solution. Right: This three-dimensional animation shows the development of the solution by assuming the wave parameter $\lambda=1$ according to the similarity transformation $\xi=x-t$; therefore the peakon moves with unit velocity in the left direction.

\section{Example 3: A nonlinear evolution equation of the fourth order}

The scaled rare studied equation e.g. (Huber, 2006; Huber, 2008) in (1+1) dimension under consideration is given by:

$$
\frac{\partial^{2} u}{\partial t^{2}}+\frac{\partial^{4} u}{\partial^{3} x \partial t}+3 \frac{\partial^{2} u}{\partial x^{2}} \frac{\partial^{2} u}{\partial x \partial t}=0, u=u(x, t), u \in C^{4}(-\infty, \infty), t>0,
$$

whereby the function $u(x, t)$ describes a wave propagation depending upon time $t$.

We seek for solutions for which $u=F(x, t)$, where $F \in C^{4}(D), D \in R^{2}$. In recent papers (Huber, 2005), the tanh-approach was used to calculate soliton solutions of (3.1). Alternatively, a new physical related application is given in (Huber, 2010b) and now we are interested here to derive new solutions.

Performing the above given steps one ends up by the nODE: $f^{(4)}+3 f^{(2)^{2}}-\lambda f^{(2)}=0, f=f(\xi)$.

Putt $h=f^{(2)}$ as new dependent variable and balancing the parameter $n$ leads to $n=2$. So the polynomial 'ansatz' of second order is suitable for our purposes

$$
h=a_{0}+a_{1} \sinh \omega+a_{2} \cosh \omega \sinh \omega+b_{1} \cosh \omega+b_{2} \cosh ^{2} \omega .
$$

One derives at an under-determined nonlinear algebraic system of equations consisting of seven equations and eight unknown. We get six solutions, four are of trivial form. The remaining two possibilities differ by the sign of the wave velocity $\lambda$ to give

$$
\begin{array}{ll}
a_{0}=\frac{1}{6}\left(31 \alpha^{2}+4 \beta^{2}+\lambda\right), & a_{1}=-\frac{10 \alpha \beta}{3}, b_{1}=0, \\
\lambda= \pm \alpha \sqrt{961 \alpha^{2}-56 \beta^{2}}, & b_{2}=-\frac{2 \beta^{2}}{3} .
\end{array}
$$

We have to require that products may not vanish commonly, e.g. $a_{i} b_{j} \neq 0$ and various combinations just as well.

Since $\alpha$ and $\beta$ are arbitrary constants in eq.(3.16), for simplicity and any loss of generality we can set $\alpha=\beta=1$ to get the following expression for the function $h=h(\xi)$ :

$$
h(\xi)=\frac{1}{6}\{N-2(\cosh [4 \arctan h[M]]-10 \cosh [4 \arctan h[M]])\},
$$


where we introduced the numerical factor $N=(33+\sqrt{905})$ and for the arguments of the inverse hyperbolic tangent functions we set $M=1+\sqrt{2} \tanh \left[\frac{\xi}{\sqrt{2}}\right]$.

Proceeding further in order to derive the original function $u=u(\xi)$ we have to integrate twice the above given function which is closely related to problems of symbolic integrations, e.g. (Bronstein, 2004).

Tangent- and trigonometric functions can be integrated by transforming them to complex logarithms and exponentials where the result contains non-elementary functions (Bronstein, 2004).

The highly complicate solution is a complex-valued function independently from the choice of the independent variable $\xi$, that is $u(\xi) \in C \forall \xi \in R \wedge \xi \in C$. We refer to the fact that the integration is possible leading to a great number of trigonometric, hyperbolic and logarithm functions.

The result of the integration also contains some complex-valued dilogarithm functions of the second kind proportional to $\sim L_{2}\left[\exp \left[2 i\left(\frac{i \xi}{\sqrt{2}}+i \operatorname{arctgh} \sqrt{2}\right)\right]\right]$, where $i^{2}=-1$ and $L_{2}($.$) means the Euler dilogarithm function.$

We do not find it worth to write down in detail this complicate function so we decided to discuss further the solution function $h(\xi)$, eq.(3.20). This function can be simplified by standard relations to transform the inverse hyperbolic tangent leading to

$$
h(\xi)=\frac{1}{6}\left\{N-2 \cosh \left[2\left[\ln \left[\operatorname{coth} \frac{\xi}{\sqrt{2}}\right]\right]\right]+\frac{20 \operatorname{cosech}^{2} \frac{\xi}{\sqrt{2}}}{1+\sqrt{2} \operatorname{coth} \frac{\xi}{\sqrt{2}}}\right\} .
$$

To exclude the singularities we have to require that the function is defined in some domain $D_{1}:=\{(h,(\xi)) \in \tilde{D}: h(\xi) \neq-\sqrt{2} \operatorname{arctgh} \sqrt{2}\}$ for the last additional hyperbolic term and further $D_{2}:=\{(h,(\xi)) \in \tilde{D}: h(\xi) \neq i \pi / \sqrt{2}\}$ for the logarithmic part. In addition we usually require $\xi \backslash\{0\}$.

In Figure 5 we show the function eq.(3.21) in some domain and we see that the function represents a typical peakon behaviour taking an infinite value as $\xi=0$. The limiting behaviour shows that we have $h(\xi) \rightarrow-\infty$ as $\xi \rightarrow 0$.

The first and the second derivatives exist but takes infinite as $\xi=0$.

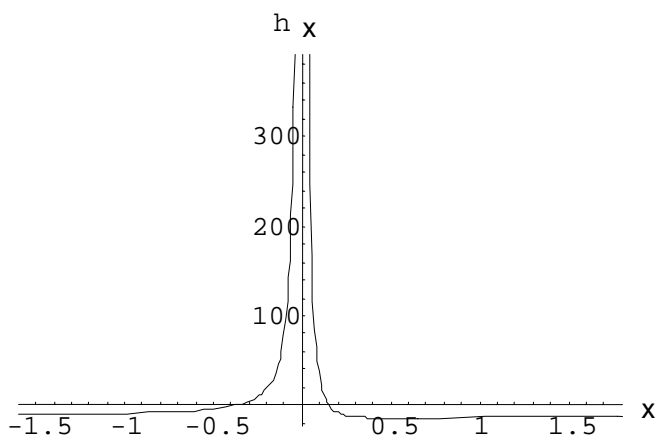

Fig.5 The new peakon solution (3.21) of the nPDE (3.17). A marked peak is observed and this solution means a new contribution to the solution-manifold of the eq.(3.17). Both for the cases $\xi \rightarrow \pm \infty$ the function takes finite.

\section{Summary and conclusion notes}

In this paper new classes of solutions of some $(1+1)$ nonlinear evolution equations of higher order by using a new algebraic approach could obtained (the case $(\mathrm{n}+\mathrm{m})$ deals analogues).

The crucial step is the assumption that the unknown solution function of any nPDE under consideration is also a solution of some ODEs which can be solved explicitly. Here in our new approach we use new formulated ODEs of the first order allowing the solution manifold explicitly expressed. 
It is known that solutions of nonlinear evolution equations can be expressed as finite series in terms of special functions, e.g. hyperbolic functions, Weierstrassian and Jacobian functions.

All these different algebraic approaches are useful if one assume the possibility to balance the nonlinear term and the highest linear term leading to a suitable number (a positive integer) in the series expression (2.3). This number may not be equal to zero since it represents the number of terms in the series expansion (obviously some equations allow $n=0$ ).

Let us now mentioned a few words to different 'ansätze' used by 'exact reduction processes' generating nODE's from nPDE (at this stage we refer to the Painlevé conjecture).

Commonly used 'ansätze' are $u(x, t)=U(x-\lambda t)$ and $u(x, t)=U(x / 3 t)^{1 / 3}$ respectively, especially for 'diffusion-like' parabolic equations. These 'ansätze' resulting from Lie group analysis (LGA) are appropriate to reduce nPDEs to nODEs in the sense of a similarity reduction.

Not only classes of travelling wave solutions occur; moreover, classes of general solutions result. However, difficulties appear in solving the relating nODE derived from the similarity reduction (for some nODEs only a numerical procedure is appropriate).

On the contrary this also represents another advantage of the given algebraic procedure since it allows generating classes of solutions in the same manner but without solving complicate nODEs explicitly.

Travelling wave solutions occur as well as complex-valued class of solutions without physical significance; but of mathematical point of view they are included in the solution-manifold and therefore indispensable.

Comparing the power of algebraic approaches with LGA methods with those from algebraic type one can say that they work as an excellent alternative tool without using any numerical methods. Apart from trivial solutions of the homogeneous nonlinear algebraic system in some cases it may happen that such systems can be solve only numerically.

Otherwise one can make use of the freedom of the appearing constants introducing as a new aspect in the assumed differential equations (2.4), (2.4a) and (2.4b).

In fact, it is clear that by increasing the number of constants (the systems might be over-determined eventually) the solubility process of the nonlinear algebraic system can therefore be influence by the user.

As a last remark one should point out the possibility in translating the approach in any computer languages.

Broadly speaking such algebraic methods are suitable to safe both the calculation time and period of vocational adjustment in any scientific as well as technical problems.

\section{References}

Bronstein M., 2004, Symbolic Integration I, Transcendental Functions, $2^{\text {nd }}$ ed., Springer

Burgers J., 1948, Adv. Appl. Mech.1, p.171

Burgers J., 1974, Asymptotic Solutions and Statistical Problems, Dodrecht, D. Reidl Publ.

Bai Ch., 2001, Exact solutions for nonlinear partial differential equation: a new approach, Phys. Lett. A 288, p.191

Cariello F., Tabor M., 1989, Painlevé expansions for nonintegrable evolution equations, Physica D 39, p.77

Cao D., 2001, New exact solutions for a class of nonlinear coupled differential equations, Phys. Lett. A 296, p.27

Chen H., H. Zhang, 2003, Improved Jacobin elliptic function method and its applications, Chaos, Solitons and Fractals, Vol. 15, p.585

Chen Y., 2003, Generalized Riccati equation expansion method and its application to the $(2+1)$ dimensional Boussinesque equation, Int. J. of Modern Physics C, Vol. 14, No. 4, p.471

Darwish A.A., 2007, Applications of algebraic method to exactly solve some nonlinear partial differential equations, Chaos, Solitons and Fractals, Vol. 33, No. 4, p.1263

Drazin P., Johnson R., 1989, Solitons: An Introduction, Cambridge University Press

Elwakil S.A., S. K. El-labany, 2003, Exact travelling wave solutions for the generalized shallow water wave equation, Chaos, Solitons, Fractals, Vol. 17, No.1, p.121

Elwakil S.A., El-labany S.K., 2004a, New exact solutions for a generalized variable coefficients 2D KdV equation, Chaos, Solitons, Fractals, Vol. 19, No. 5, p.1083

Elwakil S.A., S. K. El-labany, 2004b, Higher-order solution of an electron acoustic solitary wave via vortex electron distribution, Chaos, Solitons, Fractals, 22/1, p.13

Huang D., 2004, Exact travelling wave solutions for the Boiti-Leon-Pempinelli equation, Chaos, Solitons and Fractals, Vol. 22, p.243

Huber A., 2005, Solitary solutions of some nonlinear evolution equations, Appl. Math. and Comp., Vol. 166, No. 2, p.464

Huber A., 2006, A novel class of solutions for a non-linear third order wave equation generated by the Weierstraß transformation, Chaos, Solitons and Fractals, Vol. 28, No. 4, p.972

Huber A., 2008a, A note on new solitary and similarity class of solutions of a fourth order non-linear evolution equation, Appl. Math. and Comp. Vol. 202, p.787

Huber A., 2008b, A note on a class of solitary-like solutions of the Tzitzéica-equation generated by a similarity reduction, Physica D 237, p.1079 
Huber A., 2009, On non-linear evolution equations of higher order - the introduction and application of a novel computational approach, Appl. Math. and Comp., 215, p.2337

Huber A., 2010, The physical meaning of a nonlinear evolution equation of the fourth order relating to locally and non-locally supercritical waves, Int. J. Engineering, Science and Technology, Vol.2, No.1, p.70

Huber A., 2010, A classical and non-classical symmetry analysis of variant Thin Film Equations, Int. J. Engineering, Science and Technology, (in press)

Khuri S.A., 2007, Traveling wave solutions for nonlinear differential equations: A unified ansätze approach, Chaos, Solitons and Fractals, Vol. 32, No. 1, p.252

Nickel J., 2007, Travelling wave solutions to the Kuramoto-Sivashinsky equation, Chaos, Solitons and Fractals, Vol. 33, p.1376

Parkes E.J., Duffy B. R., 1997, Travelling solitary wave solutions to a compound KdV-Burgers equation, Phys. Lett. A 229, p.217

Parkes E.J., Duffy B.R., 1996, An automated tanh-function method for finding solitary wave solutions to non-linear evolution equations, Computer Physics Communications, Vol. 98, p.288

Wadati M., Konno K., 1975, Relationships among inverse method, bäcklund transformation and an infinite number of conservation laws, Progr. Theoret. Phys. Vol. 53, p.419

Wang M., 1996, Exact solutions for a compound KdV-Burgers equation, Phys. Lett. A 213, p.279

Whilemsson H., 1987, Phys. Rev. A 36, p.965

\section{Biographical notes}

Dipl.-Ing. Dr. techn. Huber Alfred is a distinguished lecturer at the Institute of Theoretical Physics - Computational Physics at the Technical University Graz, Austria following his Habilitation treatise. He did his Diploma thesis titled 'Systematic in the physics of elementary particles focusing the quarkonium states' in the field of elementary particle physics at the former Institute of Nuclear Physics at the Technical University Graz, Austria. He completed his scientific education with the Doctoral programme of technical sciences at the Institute of Chemical Technology of Inorganic Compounds at the Technical University Graz, Austria subject to nuclear solid state physics and advanced electrochemistry. Thesis titled 'Synthesis and characterization of doped manganese dioxides'. Also the author has a learnt vocation for a chemical assistant at the Research Centre of Electron Microscopy at the former Technical High School Graz, Austria. He is the author of 34 articles which appeared in world-wide renowned scientific journals. His research interests are nonlinear partial differential equations (nPDE) of higher order with applications especially in physics and chemistry. The author developed several new algebraic procedures for solving nPDE. Special interests are given in classical and non-classical symmetry methods, nonlinear transformations and the application of nonlinear methods in describing electrochemical interfaces, nonlinear wave propagation and further nonlinear topics of advanced character.

Received February 2009

Accepted April 2010

Final acceptance in revised form May 2010 